\title{
Measuring the elevated temperature dependence of up-conversion in Nd:YAG.
}

\author{
RenPeng Yan, Sung Jin Yoon, Stephen J. Beecher, and Jacob I. Mackenzie
}

\begin{abstract}
We present measurement of the temperature dependence of the energy transfer up-conversion coefficient from the main upper laser level $\left({ }^{4} \mathbf{F}_{3 / 2}\right)$ of 1at.\% doped Nd:YAG. This is achieved by a very simple method employing a $\mathrm{z}$-scan technique, monitoring the transmitted power of a probe laser tuned to the main absorption peak at $808 \mathrm{~nm}$. For analysis a simple model was developed and the temperature dependence of the absorption coefficient for this absorption band, covering the temperature range from $300-450 \mathrm{~K}$, was measured. A spatially dependent two-level rate equation model is described, which simulates the relationship between the incident pump irradiance and power transmitted by the crystal, as a function of its temperature. By comparing the experimental results with the model, we obtain a value for the energy transfer up-conversion coefficient of $4.7 \pm 0.5 \times 10^{-17} \mathrm{~cm}^{3} / \mathrm{s}$, at room temperature, decreasing to $1.6 \pm 0.2 \times 10^{-17} \mathrm{~cm}^{3} / \mathrm{s}$ at $450 \mathrm{~K}$.
\end{abstract}

Index Terms - Nd:YAG, Up-conversion, absorption coefficient.

\section{INTRODUCTION}

$\mathrm{S}$ OON after the first solid-state laser was reported, Nd:YAG was demonstrated to be an exemplary gain medium, and has since become one of the workhorse gain media for many industrial, medical and scientific laser systems. Despite a relatively high quantum defect ( $25 \%$ for $808 \mathrm{~nm}$ pumping and lasing on the strongest emission line at $1064 \mathrm{~nm}$ ), the spectroscopic properties of this transition enable efficient operation in medium average power systems and in recent years even into the $\mathrm{kW}$ regime $[1,2]$. The lower gain transition emitting around $946 \mathrm{~nm}$, could potentially be more efficient than the standard four-level dominant transition due to the lower quantum defect, however, suffering reabsorption losses at the laser wavelength, this laser's performance is severely affected by detrimental thermal effects in the host material and even relatively small cavity losses [3-5]. In recent years, new laser architectures as well as high radiance laser diodes have been developed that help to mitigate the thermal distortion

This work was supported in part by the UK Engineering and Physical Science Research Council (EPSRC) via grants EP/H005412/1 and EP/J008052/1. RenPeng Yan would like to acknowledge the China Scholarship Council for financial support, while Sung Jin Yoon would like to thank Charm Engineering Inc.(Korea) for their financial support via a PhD scholarship.

The authors are with the Optoelectronics Research Centre, University of Southampton, Hampshire SO17 1BJ, U.K. (e-mail: ry2g13@soton.ac.uk; sjy1g12@orc.soton.ac.uk; S.J.Beecher@ soton.ac.uk; jim@orc.soton.ac.uk).

RenPeng Yan is also with the National Key Laboratory of Science and Technology on Tunable Laser, Harbin Institute of Technology, Harbin 150080, China. effects, including composite end-capped bulk crystals, thin-disks, single crystal fibers, and the planar waveguide [6-10]. Thanks to intense pumping offered by ever improving diode lasers, a high population inversion is possible at relatively low powers, which can enable efficient operation of this quasi-four-level laser [11]. However, despite the potential of available pump sources, improvement in the $946 \mathrm{~nm}$ laser performance beyond $15 \mathrm{~W}$ [8] in a bulk composite-rod is still restricted by the heat generated during laser operation [5].

One potentially significant parameter that adds to the non-radiative de-excitation of the upper laser-level during laser operation is energy transfer up-conversion (ETU). The ETU process involves two $\mathrm{Nd}^{3+}$ ions in their excited meta-stable state, ${ }^{4} \mathrm{~F}_{3 / 2}$, one of which receives the stored energy of the other and is subsequently excited to a higher energy state $\left({ }^{4} \mathrm{G}_{5 / 2},{ }^{4} \mathrm{G}_{7 / 2}\right.$, or $\left.{ }^{2} \mathrm{G}_{9 / 2}\right)$, while the other one is returned to a lower state $\left({ }^{4} \mathrm{I}_{15 / 2}\right.$, ${ }^{4} \mathrm{I}_{13 / 2}$, or ${ }^{4} \mathrm{I}_{11 / 2}$ ) respectively [5]. Depending upon the strength of the ETU processes, this can have a detrimental effect on laser performance, as an additional source of heat, increased cavity losses, and by reducing the population inversion thus lowering the achievable gain, potentially catastrophic for the weaker $946 \mathrm{~nm}$ transition.

Whilst there are many papers studying the influence of ETU on quasi-four-level laser performance (more commonly referred to as a quasi-three-level), both experimentally and in simulation [5, 12, 13], the magnitude of the reported rate-equation ETU coefficient for $\mathrm{Nd}^{3+}: \mathrm{YAG}$ varies significantly. Values from $5 \times 10^{-17}$ to $3 \times 10^{-16} \mathrm{~cm}^{3} / \mathrm{s}$ are to be found in the literature [14-16], and as such the actual impact on performance can be difficult to ascertain with certainty. Moreover, as there is evidence that the ETU coefficient has a temperature dependence in lightly doped Er:YAG [17], it is reasonable to assume that it will also be evident in Nd:YAG. Considering that the temperature of the gain crystal increases due to the waste heat deposited during the pumping cycle, it is important to determine the actual temperature dependence of the ETU coefficient to fully appreciate its effect on the performance of the weaker lasing transitions.

In this work, we investigate the temperature dependence, from room temperature up to $450 \mathrm{~K}$, of the macroscopic up-conversion coefficient for the ${ }^{4} \mathrm{~F}_{3 / 2}$ meta-stable energy level, based upon an accurate determination of the $808 \mathrm{~nm}$ absorption coefficient, and its subsequent saturation as a function of the incident irradiance of a pump laser beam in a z-scan set-up. In the following, we detail the experimental setups, observed results and discuss the derived dependence of the magnitude of 
the up-conversion coefficient over the same temperature range.

\section{THEORETICAL BACKGROUND AND MODEL}

The z-scan technique gives accurate control of the incident probe irradiance, simply by scanning a "slowly" converging/diverging laser beam through the sample under test [17]. However, to fully appreciate the change in transmission of the probe laser, it is necessary to know the small signal transmission at the wavelength of interest and temperature of the sample.

For most of today's interesting laser crystals, it is known that the spectroscopic properties are strongly temperature dependent [18]. While the temperature dependence of Nd:YAG's emission cross section has been well documented [18], the details for the equivalent conditions for the pump absorption lines are not readily available. As such to enable an accurate measurement of the up-conversion coefficient it was first necessary to undertake a comprehensive study of the absorption properties of the crystal as it was heated from $300 \mathrm{~K}$ to $450 \mathrm{~K}$.

\section{A. Measurement of absorption coefficient}

To measure the absorption coefficient of our Nd:YAG crystal, we employed the broadband Amplified Spontaneous Emission (ASE) from sub-threshold diode lasers and the Beer-Lambert law. The law relates to the absorption of light as it passes through the material.

$$
A=-\log T, T=\frac{I_{t}}{I_{o}}
$$

where $I_{t}$ is unabsorbed irradiance, $I_{0}$ is the probe laser incident irradiance. $A$ is the absorbance of the crystal, while $T$ is the transmittance.

$$
T=T_{1} T_{2} \exp \left(-\alpha_{a} l_{c}\right)
$$

where $T_{1}$ and $T_{2}$ are the transmission values for the $1^{\text {st }}$ and $2^{\text {nd }}$ crystal faces, $\alpha_{a}$ is the absorption coefficient, $l_{c}$ is the path length of the pump light through the crystal. Etalon effects can be ignored due to the spectral bandwidth of the pump source and the low quality factor of the "cavity" formed between the crystal end faces. Substituting Eq. (2) into Eq. (1), gives the simple expression

$\alpha_{a}=\frac{A+\log \left(T_{1} T_{2}\right)}{l_{c} \log (e)}$

The samples used in the following experiments had been anti-reflection (AR) coated on both entrance and exit faces for a laser wavelength of $1064 \mathrm{~nm}$, and as such it was necessary to correct the measured transmission for the coatings over the spectral response of the probe light. All absorption measurements were conducted under low pump irradiances to avoid ground state depletion or excited state transfer effects.

\section{B. Z-scan measurement for ETU coefficient}

In contrast to the absorption measurements, to determine the ETU coefficient the incident irradiance must range from values much lower than the saturation irradiance (intensity) of the laser medium, to one that is comparable, if not higher than it. At these high irradiance levels, depletion of the ground state ions increases the pump transmission, however, ETU counteracts this by effectively depopulating the upper level and reducing its lifetime, equivalent to increasing the saturation irradiance condition. A suitably configured z-scan measurement provides an incidence irradiance that can be scaled over several orders of magnitude, without requiring this degree of sensitivity or linearity from the detectors and associated digitization equipment. Consequently, this method can provide a very sensitive measure of the magnitude of the ETU coefficient.

Besides ETU, cross relaxation also depopulates the upper level population via interactions between neighboring excited and ground state ions, and must be considered as a significant de-excitation process. Due to the large energy gap between ${ }^{4} \mathrm{~F}_{3 / 2}$ and ${ }^{4} \mathrm{I}_{15 / 2}$, being at least six times larger than the maximum phonon energy of the crystal, the probability of multi-phonon non-radiative relaxation can be neglected. On the other hand, as the upper pump levels $\left({ }^{4} \mathrm{~F}_{5 / 2},{ }^{2} \mathrm{H}_{9 / 2}\right)$ is only $\sim 800 \mathrm{~cm}^{-1}$ above the meta-stable level, the excited ions rapidly ( ns timescale) relax into the energy state of interest ${ }^{4} \mathrm{~F}_{3 / 2}\left(N_{2}\right)$. As such for Nd:YAG we can treat the problem as a two-level system between the ground state $\left({ }^{4} \mathrm{I}_{9 / 2}-N_{l}\right)$ and the meta-stable level $\left({ }^{4} \mathrm{~F}_{3 / 2}-N_{2}\right)$, for which the rate equations under steady state pumping conditions, are expressed as:

$$
\begin{aligned}
& \frac{\partial N_{1}(r, z)}{\partial t}=-\frac{I_{P}(r, z)}{h v_{P}} \sigma_{a b s} N_{1}(r, z)+\frac{N_{2}(r, z)}{\tau_{0}}+W_{E T U} N_{2}(r, z)^{2} \\
& +W_{C R} N_{1}(r, z) N_{2}(r, z) \\
& \frac{\partial N_{2}(r, z)}{\partial t}=\frac{I_{P}(r, z)}{h v_{P}} \sigma_{a b s} N_{1}(r, z)-\frac{N_{2}(r, z)}{\tau_{0}}-W_{E T U} N_{2}(r, z)^{2} \\
& -W_{C R} N_{1}(r, z) N_{2}(r, z)
\end{aligned}
$$

where $N_{l}$ and $N_{2}$ represent the population density in the lower $\left({ }^{4} \mathrm{I}_{9 / 2}\right)$ and upper $\left({ }^{4} \mathrm{~F}_{3 / 2}\right)$ manifolds, $\tau_{0}$ is the intrinsic radiative lifetime for Nd:YAG, $\sigma_{a b s}$ is the effective absorption cross section from the ground state manifold $N_{l}, h v_{P}$ is the pump photon energy, $W_{E T U}$ is the energy transfer up-conversion coefficient, $W_{C R}$ is the cross relaxation coefficient, and $I_{P}(r, z)$ is the spatial pump density characterized by:

$\frac{d I_{P}(r, z)}{d z}=I_{P}(r, z)\left(-\sigma_{a b s} N_{1}(r, z)\right)$

The model places emphasis on the overall influence of ETU and cross relaxation on the population inversion and pump saturation. Actually, these processes involve static and migration-assisted energy-transfer mechanisms and have different time-dependencies for the down- and up-conversion processes [19]. Since these processes are fast compared with the upper state lifetime, we have used the simplified expressions to describe the steady state condition. Note however, care must be exercised in describing Nd:YAG by these simple macroscopic rates, as fast direct-transfer processes can be missed as discussed by Lupei and Lupei [19]. To accommodate the reduced quantum efficiency observed in $\mathrm{Nd}: Y A G$, compared to that expected from the measured with respect to the intrinsic lifetime, we have utilized a cross-relaxation coefficient of $W_{C R}=0.7 \times 10^{-17} \mathrm{~cm}^{3} / \mathrm{s}$ obtained from an emission quantum efficiency of 0.8 for 1 at. $\% \mathrm{Nd}$ :YAG at room temperature [20]. Experimental measurements of the 
fluorescence lifetime performed over the temperature range from $300 \mathrm{~K}$ to $450 \mathrm{~K}$ show no variation, to within experimental errors, which confirms that the concentration dependent lifetime is invariant with temperature. We have assumed, for this work, a unique value for $W_{C R}$, as the mean energy of the manifolds involved $\left({ }^{4} \mathrm{~F}_{3 / 2}-{ }^{4} \mathrm{I}_{15 / 2},{ }^{4} \mathrm{I}_{9 / 2}-{ }^{4} \mathrm{I}_{15 / 2}\right)$ show a similar red shift with increasing temperature [21-22], and as such, while the line-shapes may change their integrals and overlap should remain reasonably constant.

The pump irradiance incident on the crystal is defined as a Gaussian distribution and the model assumes an effective beam area $\left(A_{\text {eff }}\right)$ averaged over the crystal length, and recalculated for each z-scan position, using Gaussian beam propagation theory. $A_{e f f}=\frac{\pi}{l_{c}} \int_{0}^{l_{c}} \omega^{2}(z) \mathrm{d} z$

At each z-position a radial mesh is defined consisting of 50 rings and 20 longitudinal segments, the outer diameter was changed for each z-position and set to be 4 times the effective beam radius, determined using (7). By numerically solving Eq. (4)-(6) for a fixed pump power and a range of beam radii, equivalent to those created when scanning the beam through the sample, the transmitted power can be calculated. Fig. 1 shows the expected transmission of our $3.2 \mathrm{~mm}$ long Nd:YAG crystal with a doping concentration of $1.33 \times 10^{20}$ ions $/ \mathrm{cm}^{3}$, versus the incident pump irradiance, at room temperature, with and without ETU effects. Comparing the case without ETU to the lowest reported value $5 \times 10^{-17} \mathrm{~cm}^{3} / \mathrm{s}$, the pump transmission would increase by almost a factor of two, when the peak on-axis incident pump irradiance reached $50 \mathrm{~kW} / \mathrm{cm}^{2}$, nearly four times higher than the saturation irradiance of the material. ETU acts to reduce the change in transmission as a function of the incident irradiance so can be used as a fitting parameter between the modelled and experimentally measured transmitted power.

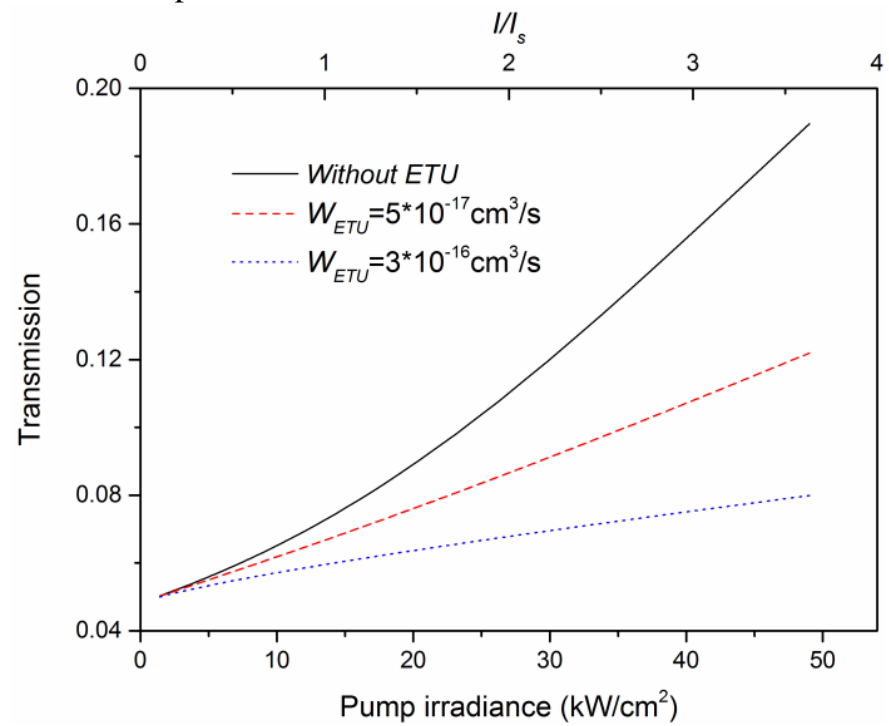

Fig. 1. Modelled $808 \mathrm{~nm}$ transmission through a $3.2 \mathrm{~mm}$ 1at $\%$ doped Nd:YAG crystal versus incident pump irradiance, referenced to the room temperature saturation irradiance.

\section{MEASUREMENT OF THE TEMPERATURE DEPENDENT ABSORPTION COEFFICIENT}

\section{A. Experimental setup}

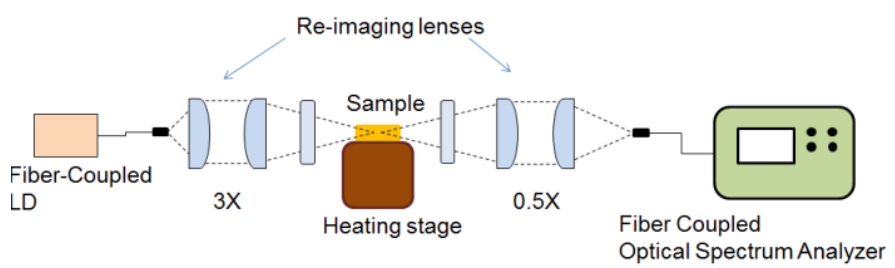

Fig. 2. Set-up for measuring absorption coefficient

The experimental setup for the absorption coefficient is shown in Fig.2. The crystal and copper $(\mathrm{Cu})$ mount was heated by a resistor in thermal contact with the mount. With this configuration the crystal temperature could be tuned from $300 \mathrm{~K}$ to $450 \mathrm{~K}$ to investigate the temperature dependence of the absorption coefficient from the ${ }^{4} \mathrm{I}_{9 / 2}(\mathrm{Z})$ ground state to the ${ }^{4} \mathrm{~F}_{5 / 2}$ and ${ }^{2} \mathrm{H}_{9 / 2}(\mathrm{~S}) \mathrm{Nd}^{3+}$ excited states, using the nomenclature of [23]. To obtain an optimum signal to noise ratio we used an AR coated $3.2 \mathrm{~mm}$ long 0.97 at. $\%( \pm 0.05 \%)$ Nd:YAG rod, wrapped in indium foil and held in contact with the $\mathrm{Cu}$ mount via a spring loaded clamp. Utilizing the broadband ASE of a fiber-coupled diode laser (LIMO60-F200-DL808) just below its threshold current $(<6.7 \mathrm{~A})$, we obtained an ASE spectrum that covered a wavelength range of $\sim 40 \mathrm{~nm}$ about the main pump band of interest, i.e. $780 \mathrm{~nm}$ to $820 \mathrm{~nm}$. With this setup we measured $10 \mathrm{~mW}$ of optical power exiting the $\varnothing 200 \mu \mathrm{m}$, NA 0.22 fiber core. A $3 \mathrm{X}$ telescope arrangement re-imaged the fiber facet producing a $600 \mu \mathrm{m}$ diameter focal spot in the crystal. Light passing through the sample was then re-imaged into a fiber patch cord $(\varnothing 300 \mu \mathrm{m}$ NA 0.22) coupled to an optical spectrum analyzer (OSA) (ANDO AQ6317B). The ASE spectrum was measured with and without the crystal in place, with a resolution of $0.1 \mathrm{~nm}$ and the absorption spectrum calculated from the difference. In the calculations to determine the absorption coefficient, the measured transmission data were corrected for the transmission of the optical coatings and set-up losses via the out of band transmission values and background measurements.

\section{B. Results and discussion}

Fig. 3 shows the derived effective absorption coefficient $\left(\alpha_{a}\right)$ spectra for Nd:YAG at various temperatures between $300 \mathrm{~K}$ and $450 \mathrm{~K}$. The neodymium concentration in the crystal was confirmed by comparing it with several other crystals of different lengths with the same specified doping level. 


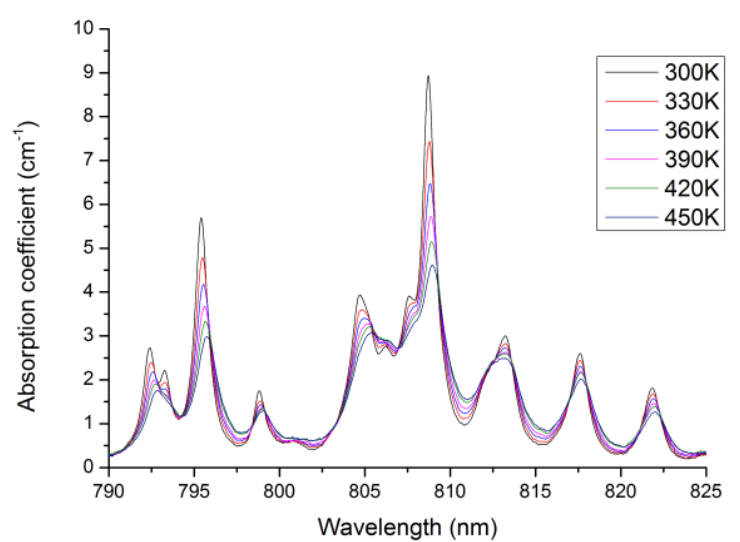

(a)

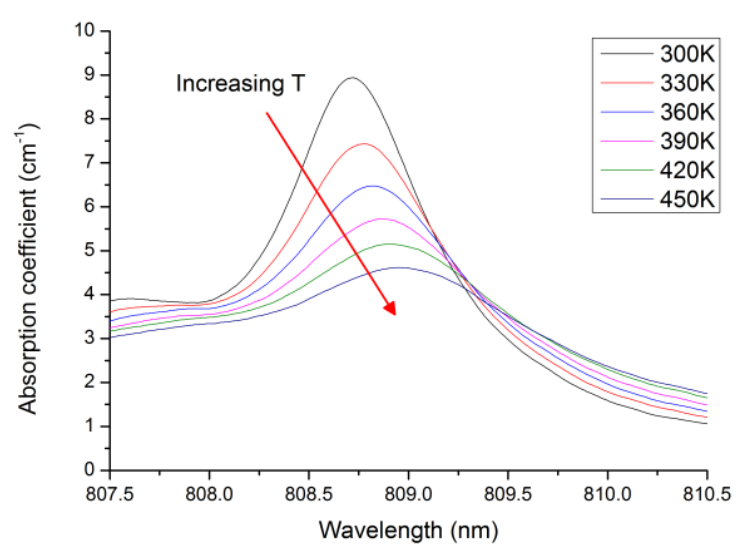

(b)

Fig. 3. Measured absorption coefficient, $300 \mathrm{~K}$ to $450 \mathrm{~K}$ (a) $790 \mathrm{~nm}-825 \mathrm{~nm}$ and (b) enlarged $808 \mathrm{~nm}$

At room temperature (RT) the maximum value for $\alpha=8.9+/-0.1 \mathrm{~cm}^{-1}$ was observed at a wavelength of $808.7 \pm 0.1 \mathrm{~nm}$. With increasing temperature, the amplitude decreases whilst the bandwidth broadens in equal measure, as shown in Fig.3. The peak of the absorbance reduced to $4.6 \pm 0.1 \mathrm{~cm}^{-1}$ at $450 \mathrm{~K}$, this is nearly a 2-fold decrease with respect to RT. This absorption peak around $808 \mathrm{~nm}$ consists of two transition lines $\mathrm{Z}_{1} \rightarrow \mathrm{S}_{1}, \quad \mathrm{Z}_{3} \rightarrow \mathrm{S}_{4}$ according to the nomenclature of Carlson and Dieke [23]. Due to the temperature rise of $150 \mathrm{~K}$, the population in the $\mathrm{Z}_{1}$ Stark level decreased by $\sim 80 \%$, while for $\mathrm{Z}_{3}$ the population increased $\sim 15 \%$, with respect to their RT condition. The combined full width half maximum (FWHM) bandwidth increases from $\sim 1 \mathrm{~nm}$ at RT to $1.4 \mathrm{~nm}$ at $330 \mathrm{~K}$, and at higher temperatures the absorption peak is so broad that it is not possible to easily define a FWHM as it starts to merge with adjacent transitions. The peak wavelength was red-shifted by $0.2 \mathrm{~nm}$ over the measured temperature range, as shown in Fig.3(b), in line with most other transitions as reported by Kushida [21].

\section{Polynomial fitting for numerical modelling}

The spectrum of our Ti:Sapphire laser (Model: 3900S, Spectra-Physics Laser, Inc.), used for the z-scan measurements, was measured to be $<0.1 \mathrm{~nm}$, and consequently being much

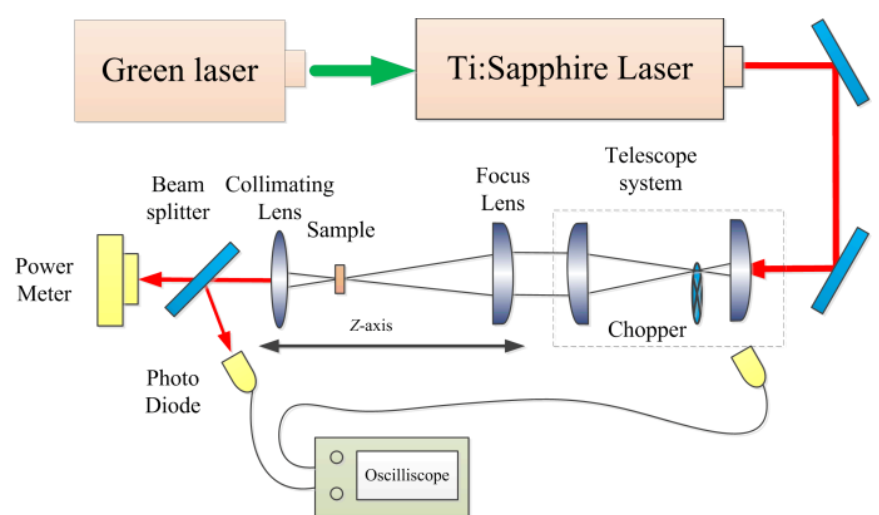

Fig. 5. Schematic of z-scan experimental setup

narrower than the bandwidth of our pump band, the absorption coefficient simply follows the peak amplitude of $\alpha_{a}$. For each new crystal temperature the emission wavelength of the Ti:sapphire pump laser was tuned to maximize the absorption, accounting for the red-shifting peak. Considering the data of Fig. 3, we have calculated the value of $\alpha_{a}$ for the temperature range of $300 \mathrm{~K}$ to $450 \mathrm{~K}$. Eq.(12) represents a polynomial fit, which can describe the temperature dependent absorption coefficient, $\alpha_{a}$;

$\alpha_{a}(T)=e_{o}+e_{1} T+e_{2} T^{2}$

Where $e_{0-} e_{2}$ are the polynomial coefficients and $T$ is the crystal temperature. A $2^{\text {nd }}$ order polynomial was used to represent the experimental data covering most of the useful temperature range for laser operation, as this gave the best fit for the measured temperature range, found to be within the experimental error range $( \pm 2 \%)$.

\begin{tabular}{cc} 
& TABLE I $\alpha_{\mathrm{a}}$ FOR ND:YAG $(300 \mathrm{~K}$ TO 450K) \\
\hline$e_{0}$ & 32.2 \\
\hline$e_{1}$ & -0.112 \\
\hline$e_{2}$ & $9.13 \mathrm{e}-5$ \\
\hline
\end{tabular}

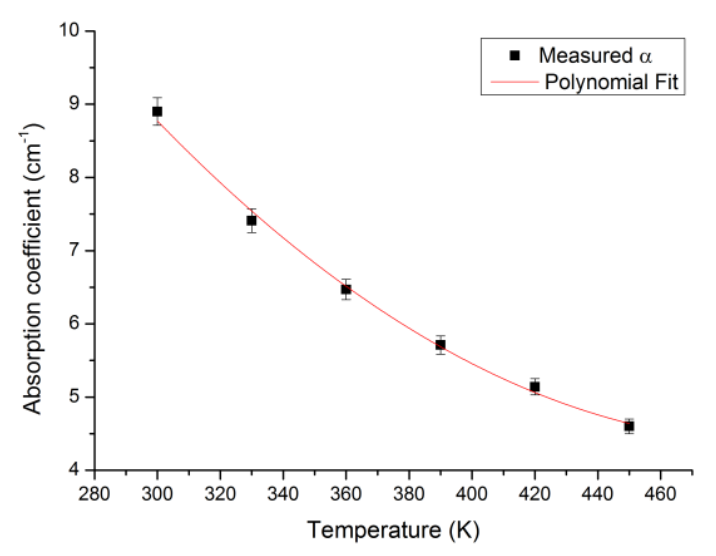

Fig. 4. Temperature dependence of the small signal peak absorption coefficient at $808 \mathrm{~nm}$ in 1 at $\%$ doped Nd:YAG between $300 \mathrm{~K}$ and $450 \mathrm{~K}$

\section{Z-SCAN MEASUREMENT OF ETU COEFFICIENT}

A. Experimental setup for the z-scan measurement 
The schematic of the z-scan experimental setup is shown in Fig.5. The output beam of the continuous-wave Ti:sapphire pump source was expanded with a telescope system $\left(f_{1}: f_{2}=30 \mathrm{~mm}: 200 \mathrm{~mm}\right)$, then mechanically chopped at the focus of the telescope. A single aperture disc was rotated at a few tens of $\mathrm{Hz}$ to generate pulses with a duty cycle of $10 \%$ and duration of $\sim 2.4 \mathrm{~ms}$. This pulse duration was long enough for the inversion density to reach steady state and the duty cycle low enough to ensure minimal additional heating of the sample considering our few hundred $\mathrm{mW}$ average power available. It should be noted that the expected temperature rise within a single pulse is calculated to be $\sim 0.1 \mathrm{~K}$, not significant in terms of the temperature range investigated. A focusing lens of $f=300 \mathrm{~mm}$ was mounted to an electronically-controlled translation stage (Stackshot, Cognisys Inc.) to change the beam size in the laser crystal. Using this translation stage the pump irradiance could be adjusted precisely through the accurate control of the focal position with respect to the sample. During the scan, the change in pump transmission was measured by collecting the light passing through the sample with a collimating lens, also mounted on the translation stage, whereby the light was attenuated using an uncoated wedge. We passed $\sim 92 \%$ of the beam directly to a power meter (13PEM100, Melles Griot Inc.), while one surface reflection $(\sim 4 \%)$ was redirected to a silicon photodiode. A digital oscilloscope (MSO6104A, Agilent Technology Inc.) was used to record the amplitude of the transmitted signal. The value of the power without the crystal was recorded as a reference level. To determine the irradiance level at each z-position, the laser beam size was measured by a beam profiler (Nanoscan II, OPHIR Inc.) placed at the location of the sample, as the lens pair were translated along the $z$ axis.

Fig. 6 shows the measured second moments beam radii as a function of longitudinal position along the beam propagation path. By fitting the Gaussian beam propagation expression to the data, the beam waist radii and the beam quality are deduced with respect to the coordinate system of the optical table. The beam propagation parameter, in both axes, was measured to be

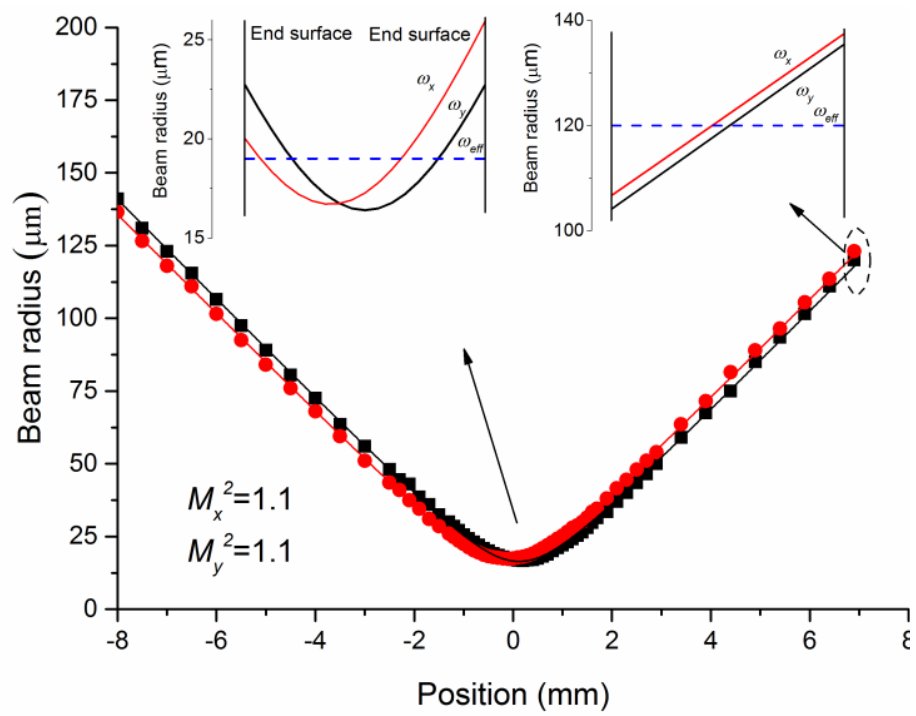

Fig. 6. Measured beam radii and the calculated effective beam radius as a function of position along the pump propagation path.
$M^{2}=1.1$, while the beam waist radii were $\omega_{x}=16.7 \pm 0.2 \mu \mathrm{m}$ and $\omega_{y}=16.4 \pm 0.2 \mu \mathrm{m}$. Therefore the confocal parameter for this beam was slightly longer than the crystal length, which is close to the limit when considering the change in beam size throughout the sample and may introduce a slight asymmetry in the transmission response depending upon whether the pump beam is converging or diverging through the crystal. Two insets in Fig. 6, illustrate the actual beam radii versus the calculated effective beam radius derived from Eq. (7). This beam focusing condition was used for the elevated temperature experiments to be discussed at the end of the next part. However, in addition a RT experiment was carried out initially with a slightly larger beam waist, $\omega=20.6 \pm 0.3 \mu \mathrm{m}$, giving a confocal parameter nearly twice that of the sample length. Fortunately the resulting outcome was the same at RT in both cases, demonstrating that the beam conditions did not adversely affect the measurement technique.

\section{B. Experimental results and discussion}

As shown in Fig. 7, the RT crystal transmission at $808 \mathrm{~nm}$ versus the sample position, with respect to the focus, presents a peak where the incident pump irradiance is highest. It is also shown that the simulated maximum crystal transmission without considering ETU overestimates the observed value by $\sim 50 \%$ as the pump irradiance reaches its maximum value. However, considering the three decays channels detailed in Eq. (4-5), at the highest pump irradiance the modelled peak transmission decreases to $\sim 12 \%$, in line with that measured for an ETU coefficient of $4.7 \pm 0.5 \times 10^{-17} \mathrm{~cm}^{3} / \mathrm{s}$, for our $\sim 1.0$ at $\%$ $\mathrm{Nd}: Y A G$ at room temperature. This is in good agreement with the value reported by Guy et al. [14], and significantly lower than that reported by others $[15,16]$. In simulation, the maximum transmission is quite sensitive to the magnitude of the ETU coefficient, providing a low range of potential values for this fitting parameter. Moreover, the simulation and the experimental data show excellent agreement over the whole z-scan range, which provides a high level of confidence in the accuracy of the measurement.

To compliment this analysis, we also recorded the temporal waveform of the pump, before and after the crystal. Comparing the two pulse profiles we could determine the temporal dependence of the bleaching effect for different locations (pump irradiance levels), several of which are presented in Fig. 8. The steady-state condition of the pulse matches very well the modelled waveform, supporting the observations in Fig. 7. For the highest pumping irradiance however, there is a slight difference between observation and simulation in the leading edge of the pump waveform, where Fig. 9 shows that at the beginning of the incident pump pulse the rate of ground state bleaching in experiment is faster than in the simulation. This is probably due to the simplified "steady-state" assumptions made to form Eqs. (4) and (5) and the equal rates for the up- and down-conversion processes implied, which is shown not to be the case by Lupei [19], and/or the result of ignored higher order ETU processes and bottlenecking states [24-25]. Further investigation of these cross relaxation and nonlinear mechanisms will be undertaken in the near future. 
When the sample was heated in the resistive oven to higher temperatures the resulting change is pump power transmission was observed to change as shown in Fig. 10.

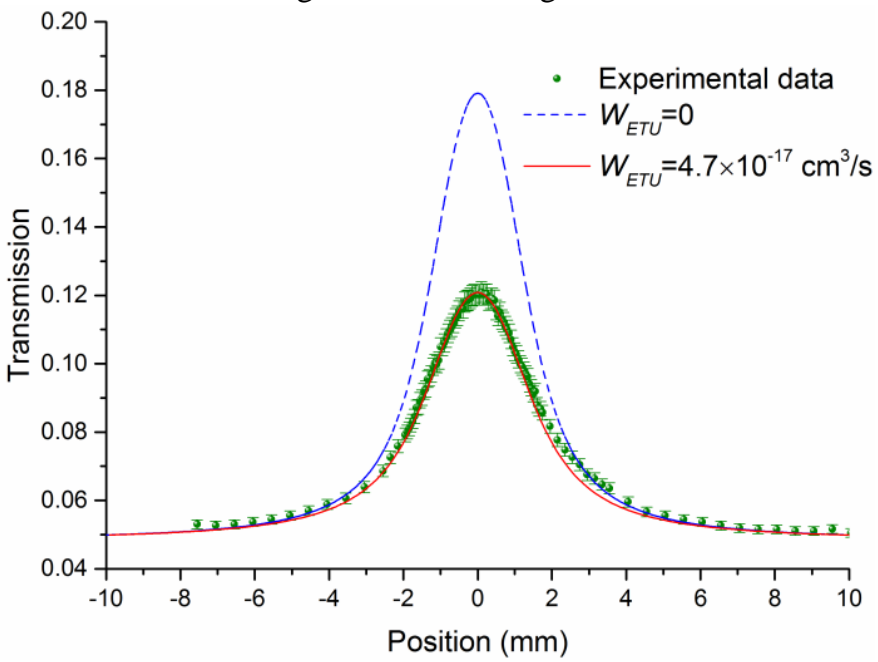

Fig. 7. Transmission of Nd:YAG crystal at RT, when varying the z-position of the focusing lens system, about the focal point at zero.

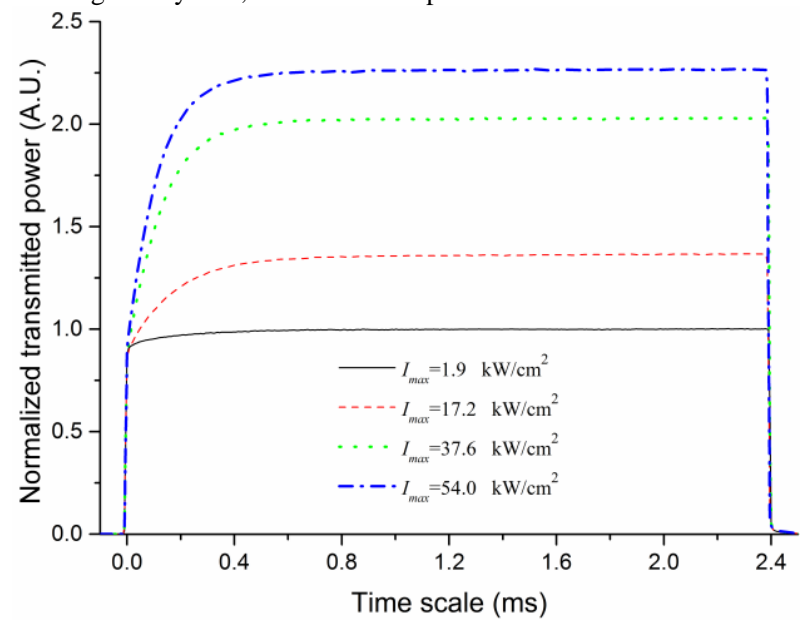

Fig. 8. RT transmitted power temporal waveforms at different incident pump irradiance levels.

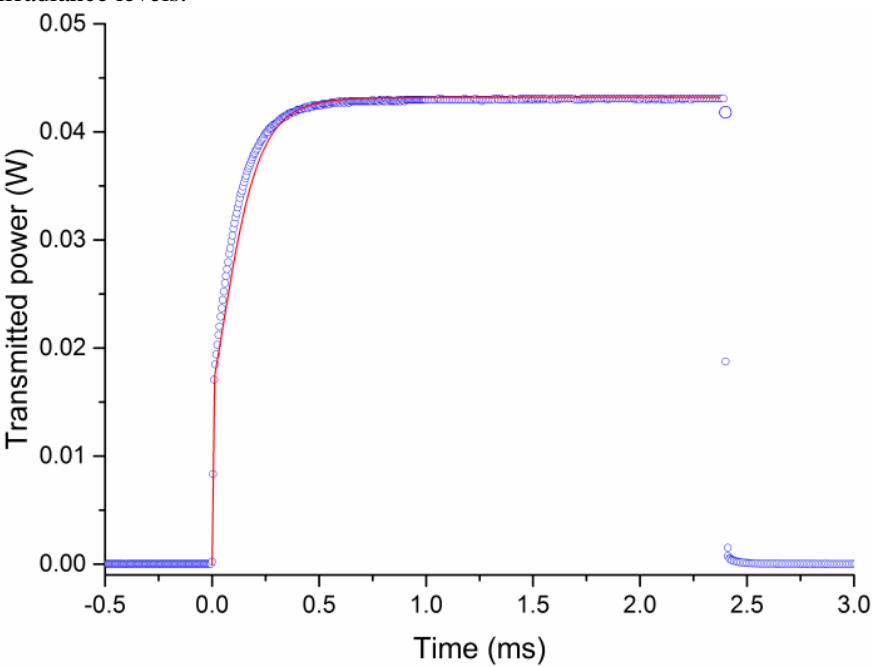

Fig. 9. RT temporal transmission variation of Nd:YAG at the maximum incident irradiance. (“o”: experimental data; “-”": simulation.)

Fig. 10 presents the experimental data and simulation results at several different temperatures from $300 \mathrm{~K}$ to $450 \mathrm{~K}$. The background transmission increases due to the reduction of the absorption coefficient as discussed earlier. At higher temperatures some instability in transmission was evident and attributed to air turbulence and localized variable cooling of the crystal facets, notwithstanding, the simulation results are in very good agreement with the experimental data, simply fitting the ETU coefficient for each respective temperature, as presented in Fig.11. It shows a nearly linear dependence of ETU coefficient on the temperature. The magnitude of ETU coefficient decreases from $4.7 \times 10^{-17} \mathrm{~cm}^{3} / \mathrm{s}$ at $300 \mathrm{~K}$ according to the equation $W_{E T U}=13.3-0.037 * T+0.000024 * T^{2}$, up to the maximum temperature investigated, $450 \mathrm{~K}$. Note in Fig. 7 and 10 there is minor skewing of the data about the peaks which may be due to the slight astigmatism in the pump beam or the asymmetry induced due to a converging or diverging beam over the crystal length, as mentioned previously.

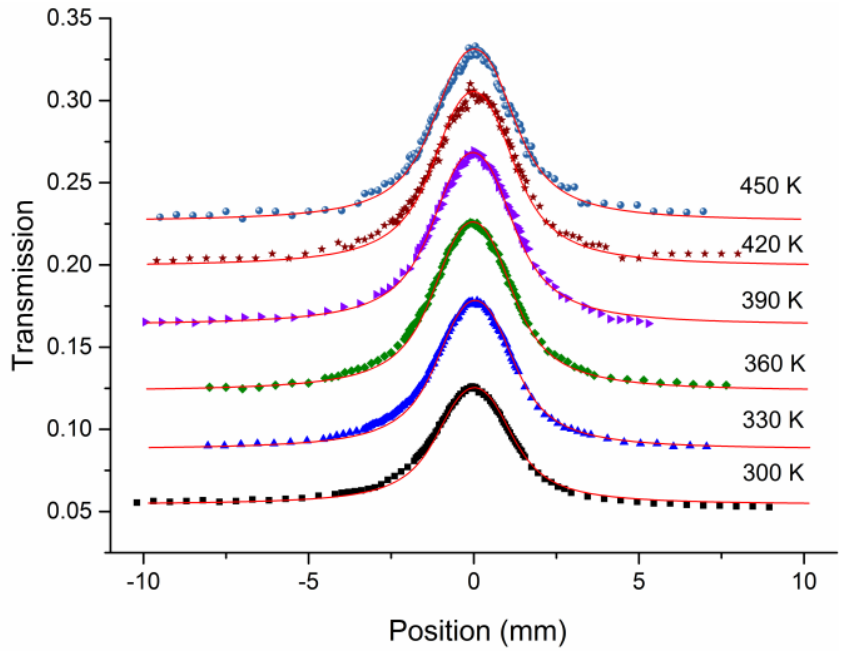

Fig. $10 \mathrm{Nd}$ :YAG crystal transmission at $808 \mathrm{~nm}$ versus z-scan position relative to the focus at different temperatures

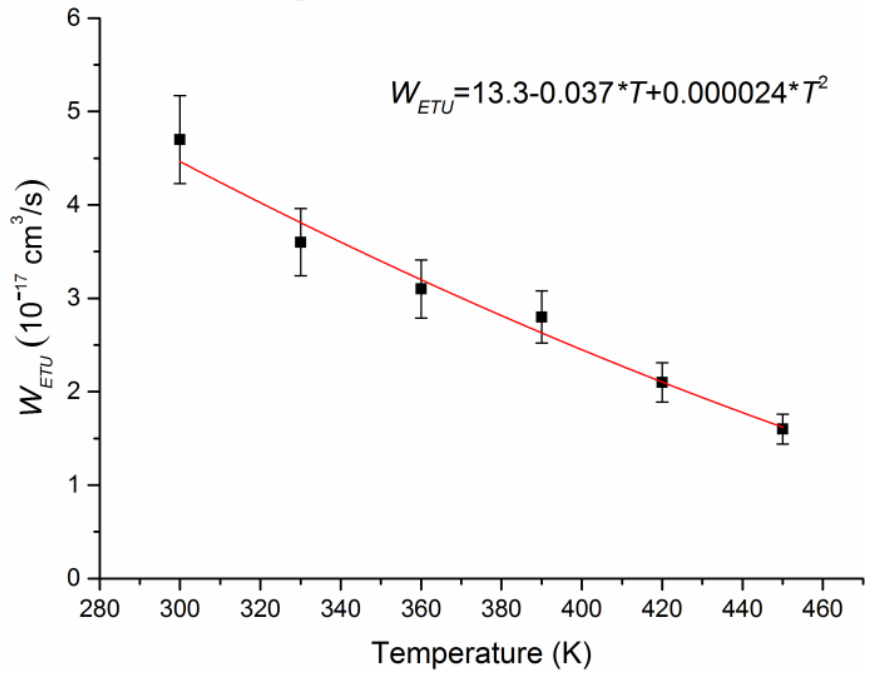

Fig. 11. The temperature dependence of the ETU coefficient for Nd:YAG

Clearly it can be seen that with increasing temperature the ETU coefficient is decreasing. Although perhaps a surprising result, considering that at elevated temperatures laser performance typically degrades and this is generally attributed to enhanced ETU, there is good evidence [22] that the potential resonances between the energy levels involved in the ETU process have a relative shift that is reducing the overlap of the respective transitions, namely: 

a) ${ }^{4} \mathrm{I}_{15 / 2}(3) \rightarrow{ }^{4} \mathrm{~F}_{3 / 2}(1),{ }^{4} \mathrm{~F}_{3 / 2}(2) \rightarrow{ }^{4} \mathrm{G}_{5 / 2}(2)$
b) ${ }^{4} \mathrm{I}_{15 / 2}(3) \rightarrow{ }^{4} \mathrm{~F}_{3 / 2}(2),{ }^{4} \mathrm{~F}_{3 / 2}(1) \rightarrow{ }^{4} \mathrm{G}_{5 / 2}(2)$
c) ${ }^{4} \mathrm{I}_{13 / 2}(3) \rightarrow{ }^{4} \mathrm{~F}_{3 / 2}(2),{ }^{4} \mathrm{~F}_{3 / 2}(2) \rightarrow{ }^{4} \mathrm{G}_{7 / 2}(4)$
d) ${ }^{4} \mathrm{I}_{13 / 2}(3) \rightarrow{ }^{4} \mathrm{~F}_{3 / 2}(1),{ }^{4} \mathrm{~F}_{3 / 2}(1) \rightarrow{ }^{4} \mathrm{G}_{7 / 2}(2)$
e) ${ }^{4} \mathrm{I}_{11 / 2}(4) \rightarrow{ }^{4} \mathrm{~F}_{3 / 2}(1),{ }^{4} \mathrm{~F}_{3 / 2}(2) \rightarrow{ }^{2} \mathrm{G}_{9 / 2}(3)$
f) ${ }^{4} \mathrm{I}_{11 / 2}(4) \rightarrow{ }^{4} \mathrm{~F}_{3 / 2}(2),{ }^{4} \mathrm{~F}_{3 / 2}(1) \rightarrow{ }^{2} \mathrm{G}_{9 / 2}(3)$.

Ultimately though, this is not the whole story, as we have observed that for diode pump sources the effective absorption coefficient increases with increasing temperature [26], whereas the emission cross section is known to decrease [18, 26], consequently the thermal power density will be increased as more power is absorbed in a shorter length, and at the same time higher inversion is required to achieve the same gain as at lower temperatures. Additionally, although there is a smaller ETU parameter, it will be offset by the need for higher population density and it is possible that a comparable thermal load (or even higher) is obtained at elevated temperatures, leading to further degradation in laser performance. Our results provide a useful dataset from which to explore the implications of ETU on laser performance, especially for the quasi-four-level Nd:YAG laser that suffers strong thermal gradients associated with the pumping cycle. There is an inherent implication that the heat deposition and gain is not a simple function of the pump distribution but is also dependent upon the crystal geometry and cooling arrangement. Compared with other neodymium doped materials [16, 25], Nd:YAG has a lower ETU coefficient, and typically better thermal properties, but the real influence of ETU on laser performance needs further investigation along with the consideration of other parameters, such as cross relaxation.

\section{CONCLUSION}

In this paper we have presented a detailed study of the temperature dependence of the $808 \mathrm{~nm}$ ground state absorption and energy transfer up-conversion coefficient in 1at.\% $\mathrm{Nd}$ :YAG. A simple expression has been derived for the peak absorption coefficient over a temperature range of $300 \mathrm{~K}$ to $450 \mathrm{~K}$, which is applicable for high spectral-purity pump sources. At $450 \mathrm{~K}$ the peak absorption coefficient near $808 \mathrm{~nm}$ is nearly halved compared to that at RT. For standard diode laser pump sources, the bandwidth of the pump source will change this characteristic equation accordingly. Where for the nominal bandwidth of a diode laser $(\sim 3 \mathrm{~nm})$ the effective pump absorption actually increases with temperature [26], which has consequences when considering the laser performance dependence upon the pump source.

Utilizing the information gathered for the absorption characteristics of Nd:YAG, we were able to employ the z-scan technique to determine the temperature dependence of the ETU coefficient, from the upper ${ }^{4} \mathrm{~F}_{3 / 2}$ laser level. As such $\mathrm{W}_{\mathrm{ETU}}$ was determined to be $4.7 \pm 0.5 \times 10^{-17} \mathrm{~cm}^{3} / \mathrm{s}$ at $300 \mathrm{~K}$ decreasing in a nearly linear fashion at a rate of $\sim 0.4 \% / \mathrm{K}$.

The information set out in this paper will underpin the fundamental understanding of some of the thermal and excited state energy transfer properties of one of the most well understood laser crystals we have today. We hope that it will also soon shed light on why the quasi-four-level $946 \mathrm{~nm}$ laser of Nd:YAG simply does not scale to higher powers in bulk-rod configurations, and will provide some direction in the choice of operation conditions for future laser systems for this transition.

\section{REFERENCES}

[1] S. Fujikawa, T. Kojima, and K. Yasui, "High-power and high-efficiency operation of a cw-diode-side-pumped Nd:YAG rod laser," IEEE J. Sel. Top. Quantum Electron. vol. 3, no. 1, pp. 40-44, Feb. 1997.

[2] K. Furuta, T. Kojima, S. Fujikawa, and J.Nishimae, "Diode-pumped 1kW Q-switched Nd:YAG rod laser with high peak power and high beam quality," Appl. Opt. vol. 44, no. 19, pp. 4119-4122, Jul. 2005.

[3] T. Y. Fan, and R. L. Byer, "Modeling and cw operation of a quasi-three-level $946 \mathrm{~nm}$ Nd:YAG laser," IEEE J. Quantum Electron. vol. 23, no. 5, pp. 605-612, May 1987.

[4] W. P. Risk, "Modeling of longitudinally pumped solid-state lasers exhibiting reabsorption losses," J. Opt. Soc. Am. B, vol. 5, no. 7, pp. 1412-1423, Jul. 1988.

[5] S. Bjurshagen and R. Koch, "Modeling of energy-transfer up-conversion and thermal effects in end-pumped quasi-three-level lasers," Appl. Opt. vol. 43, no. 24, pp. 4753-4767, Aug. 2004.

[6] J. I. Mackenzie, C. Li, and D. Shepherd, "Multi-watt, high efficiency, diffraction-limited Nd:YAG planar waveguide laser," IEEE J. Quantum Electron. vol. 39, no. 3, pp. 493-500, Mar. 2003.

[7] J. Gao, J. Speiser, and A. Giesen, "25-W diode-pumped continuous-wave quasi-three-level Nd:YAG thin disk laser," in Technical Digest of Advanced Solid-State Photonics, Vienna, Austria, Feb. 2005, paper TuB34.

[8] R. Zhou, E. Li, H. Li, P. Wang, and J. Yao, "Continuous-wave, 15.2W diode-end-pumped Nd:YAG laser operating at $946 \mathrm{~nm}$," Opt. Lett. vol. 31, no. 12, pp. 1869-1871, Jun. 2006.

[9] X. Délen, I. Martial, J. Didierjean, N. Aubry, D. Sangla, F. Balembois, and P. Georges, " $34 \mathrm{~W}$ continuous wave Nd:YAG single crystal fiber laser emitting at 946 nm,” Appl. Phys. B, vol. 104, no. 1, pp. 1-4, Jul. 2011.

[10] S. P. Ng, and J. I. Mackenzie, "Power and Radiance Scaling of a $946 \mathrm{~nm}$ Nd:YAG Planar Waveguide Laser," Laser Phys. vol. 22, no. 3, pp. 494-498, Mar. 2012.

[11] N. P. Barnes, B. M. Walsh, R. L. Hutcheson, and R. W. Equall, "Pulsed ${ }^{4} \mathrm{~F}_{3 / 2}$ to ${ }^{4} \mathrm{I}_{9 / 2}$ operation of Nd lasers," J. Opt. Soc. Am. B, vol. 16, no. 12, pp. 2169-2177, Dec. 1999.

[12] J. W. Kim, J. I. Mackenzie, and W. A. Clarkson, "Influence of energy-transfer-up-conversion on threshold pump power in quasi-three-level solid-state lasers," Opt. Express, vol. 17, no. 14, pp. 11935-11943, Jul. 2009.

[13] R. Yan, X. Yu, X. Li, D. Chen, and J. Yu, "Theoretical and experimental investigation of actively Q-switched Nd:YAG $946 \mathrm{~nm}$ laser with considering ETU effects," Appl. Phys. B, vol. 108, no. 3, pp. 591-596, Sep. 2012.

[14] S. Guy, C. L. Bonner, D. P. Shepherd, D. C. Hanna, A. C. Tropper, and B. Ferrand, "High-inversion densities in Nd:YAG: up-conversion and bleaching," IEEE J. Quantum Electron. vol. 34, no. 5, pp. 900-909, May 1998.

[15] Y. Guyot, H. Manaa, J. Y. Rivoire, R. Moncorgé, N. Garnier, E. Descroix, M. Bon, and P. Laporte, "Excited-state-absorption and up-conversion studies of $\mathrm{Nd}^{3+}$-doped single crystals $\mathrm{Y}_{3} \mathrm{Al}_{5} \mathrm{O}_{12}, \mathrm{YLiF}_{4}$, and $\mathrm{LaMgAl}_{11} \mathrm{O}_{19}$," Phys. Rev. B vol. 51, no. 2, pp. 784-799, Jan. 1995.

[16] Y. F. Chen, C. C. Liao, Y. P. Lan, and S. C. Wang, "Determination of the auger up-conversion rate in fiber-coupled diode end-pumped Nd:YAG and Nd: $\mathrm{YVO}_{4}$ crystals," Appl. Phys. B, vol. 70, no. 4, pp. 487-490, Jun. 2000.

[17] J. O. White, and C. E. Mungan, "Measurement of up-conversion in Er:YAG via Z-scan,” J. Opt. Soc. Am. B, vol. 28, no. 10, pp. 2358-2361, Oct. 2011.

[18] Y. Sato, and T. Taira, "Temperature dependencies of stimulated emission cross section for Nd-doped solid-state laser materials," Opt. Mater. Express, vol. 2, no. 8, pp. 1076-1087, Aug. 2012.

[19] V. Lupei, and A. Lupei, "Emission dynamics of the ${ }^{4} \mathrm{~F}_{3 / 2}$ level of $\mathrm{Nd}^{3+}$ in YAG at low pump intensities," Phys. Rev. B vol. 61, no. 12, pp. 8087-8098, Mar. 2000.

[20] V. Lupei, "RE ${ }^{3+}$ emission in garnets: multisites, energy transfer and quantum efficiency," Opt. Mater. vol. 19, no. 1, pp. 95-107, Feb. 2002. 
[21] T. Kushida, "Linewidths and thermal shifts of spectral lines in neodymium-doped yttrium aluminum garnet and calcium fluorophosphate," Phys. Rev. vol. 185, no. 2, pp. 500-508, Sep. 1969.

[22] A. A. Kaminskii, Crystalline Lasers: Physical Processes and Operating Schemes - Chapter2 (CRC Press, 1996)

[23] E. H. Carlson, and G. H. Dieke, "The state of the $\mathrm{Nd}^{3+}$ ion as derived from the absorption and fluorescence spectra of $\mathrm{NdCl}_{3}$ and their Zeeman effects," J. Chem. Phys. vol. 34, no. 5, pp. 1602-1609, May 1961.

[24] D. A. Zubenko, M. A. Noginov, V. A. Smirnov, and I. A. Shcherbakov, "Different mechanisms of nonlinear quenching of luminescence," Phys. Rev. B, vol. 55, no. 14, pp. 8881-8886, Apr. 1997.

[25] J. D. Zuegel and W. Seka, "Up-conversion and reduced ${ }^{4} \mathrm{~F}_{3 / 2}$ upper-state lifetime in intensely pumped Nd:YLF," Appl. Opt. vol. 38, no. 12, pp. 2714-2723, Apr. 1999.

[26] S. J. Yoon, and J. I. Mackenzie, "Implications of the temperature dependence of Nd:YAG spectroscopic values for low temperature laser operation at $946 \mathrm{~nm}$ " Proc. SPIE. 9135, Laser Sources and Applications II, 913503, May 2014

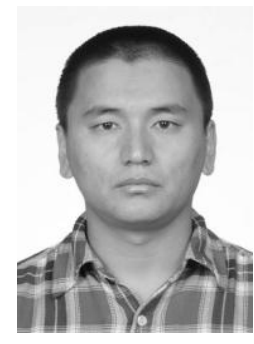

RenPeng. Yan received a B.Sc. degree in electronic technology and science Harbin Engineering University Harbin, China in 2008 and a M.S degree in physical electronics from Harbin Institute of Technology Harbin, China in 2010. He is now working toward $\mathrm{Ph}$. D. degree in physical electronics from Harbin Institute technology, Harbin, China. His research interest is frequency doubling and quasi-four-level lasers.

$\mathrm{He}$ is a visiting student in Optoelectronics Research Centre at the University of Southampton supported by China Scholarship Council.

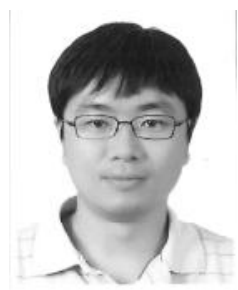

Sung Jin Yoon received B.Sc. degree in physics from the University of Auckland, Auckland, New Zealand in 2004. He is now pursuing the Ph.D degree at Optoelectronics Research Centre at the University of Southampton where he joined late 2012.

Mr Yoon worked for Charm Engineering Inc. in Korea as an engineer from 2004 until 2012, involved in development of laser micro-processing in LCD, AMOLED fields. His research topic is power scaling of quasi-four level laser.

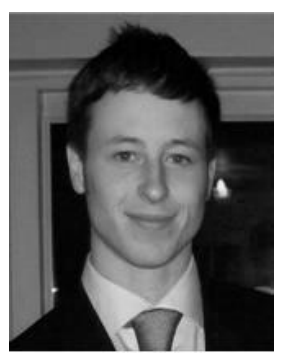

Stephen J. Beecher received a B.Sc. (Hons.) degree in physics from Imperial College London, U.K. in 2005, and a M.Sc. as a joint degree from the University of St. Andrews, U.K., and Heriot-Watt University, U.K. in 2007. He received his $\mathrm{Ph} . \mathrm{D}$. degree in physics from Heriot-Watt University in 2012. He currently works as a Research Fellow in the Planar Waveguide and Slab Lasers group, part of the Optoelectronics Research Centre at the University of Southampton. His interests are in solid state laser physics, particularly the development and application of waveguides to realize parameter combinations that are difficult to achieve with other laser geometries.

Jacob I. Mackenzie received a B.Tech. (Honors) degree in

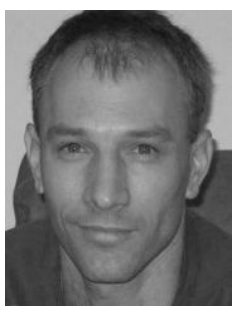
optoelectronics from Macquarie University, Sydney, Australia, in 1994. In 2003 he obtained his Ph.D. from the Optoelectronics Research Centre (ORC), University of Southampton, U.K.

Between 1995 and 1999 he was an Electro-Optic Engineer with the BAE Systems, Australia, and since 2004 a Research Fellow at the ORC. In 2004 he was a recipient of a Royal Academy of Engineering (RAE) Fellowship, and now is a Senior Lecturer and leads the Planar Waveguide and Slab Lasers (PWSL) research group in the ORC. Current research interests focus on the excellent thermal management properties afforded by the planar waveguide architecture and power-scalable solutions for new laser systems. This includes the investigation of visible, quasi-four-level, and challenging wavelength laser transitions. Dr Mackenzie is a member of the SPIE and senior member of the Optical Society of America. 